\title{
Posibilidades educativas de la realidad virtual y la realidad combinada: una mirada desde el conectivismo y la bibliotecología
}

\author{
Educational Possibilities of Virtual Reality and Combined Reality: A Glance \\ from Connectivism and Librarianship
}

Sergio Oliveros-Castro'; Claudia Núñez-Chaufleur ${ }^{2}$

\begin{abstract}
RESUMEN
La realidad virtual y la realidad combinada forman parte de las tecnologías con mayor potencial en la enseñanza y aprendizaje en las diferentes áreas del conocimiento, y han comenzado a tener un impacto significativo en el desarrollo de la sociedad de la información. El objetivo de este trabajo es analizar las posibilidades educativas de ambas para la enseñanza. El análisis se realiza desde el conectivismo, como teoría emergente del aprendizaje, la alfabetización en información y la visual literacy, como conocimientos especializados de la bibliotecología que tratan sobre el uso y reconocimiento de la información. En el artículo se ponen a disposición algunas reflexiones sobre el tema y se proponen softwares y hardwares que podrían ser aplicables al desarrollo de competencias profesionales en diferentes áreas y que, a la vez, facilitarían el aprendizaje personalizado de los/as estudiantes en base a sus capacidades e intereses.
\end{abstract}

Palabras claves: realidad virtual; realidad combinada; conectivismo; visual literacy; alfabetización en información.

\begin{abstract}
Virtual reality and combined reality are part of the technologies with the greatest potential in teaching and learning in different areas of knowledge and have begun to have a significant impact on the development of the information society. The objective of this work is to analyze the educational possibilities of both for teaching. The analysis is carried out from connectivism, as an emerging theory of learning, and information literacy and visual literacy, as specialized knowledge of Library Science dealing with the use and recognition of information. In this article some reflections on the subject are made available and some softwares and hardwares are proposed that could be applicable for the development of professional competences in different areas, and, at the same time, would facilitate student's personalized learning based on their habilities and interests.
\end{abstract}

Keywords: Virtual reality; combined reality; conectivism; visual literacy; information literacy.

\footnotetext{
${ }^{1}$ Bibliotecólogo Referencista, Universidad San Sebastián, Santiago, Chile; magíster en Educación Superior; sergio.oliveros@uss.cl

${ }^{2}$ Profesora de Educación Básica, Colegio Lafquén Montessori, Puerto Montt, Chile; claudia.nunez.ch@ gmail.com.
} 


\section{Introducción}

En su evolución, el desarrollo de las tecnologías ha pasado por diferentes etapas y ha ido, de esta manera, determinando el ritmo de los cambios en la sociedad. En términos generales, podemos distinguir la etapa de las tecnologías primitivas o de subsistencia (prehistoria a 7000 a. C.), la de las tecnologías artesanales o manufactureras (7000 a. C. a 1650), la de las tecnologías mecanizadas o industriales (1650 a 1950), la de las tecnologías de automatización o de punta (1950 a 1990) y, finalmente, la de las llamadas tecnologías éticas o de sostenimiento (1990 a generaciones futuras) (Belli y López, 2008).

Las dos últimas etapas se destacan porque en ellas han ocurrido gran cantidad de avances, descubrimientos y transformaciones, en todas las áreas de la humanidad, en periodos muy reducidos (Gay y Ferreras, 2016). En el periodo de posguerra, asistimos a una explosión de descubrimientos científicos derivados de la cibernética, la física y la electrónica, los cuales dieron como resultados avances técnicos como las computadoras portátiles y la masificación de la internet a nivel global (Castells, 1996).

Estos logros produjeron cambios como el aumento de la importancia de los servicios por sobre la producción, y el rompimiento de lo sincrónico y lo local (Tobón, Guzmán, Hernández y Cardona, 2015). Estos cambios se caracterizan por su constante desarrollo y por el hecho de que en ellos las tecnologías se incorporan en las interacciones sociales, estimulando la expansión de la idea de realidad, gracias a los dispositivos externos que permiten a las personas acceder a diferentes medios de información en instantes, comprimir las distancias y tiempos de los actos comunicativos y, además, facilitar procesos de enseñanza-aprendizaje mediante nuevas didácticas tecnológicas (Gay y Ferreras, 2016).

Es en este contexto que la aparición de las tecnologías de realidad virtual y realidad combinada se presentan como medios para el mejoramiento de los procesos educativos, pudiendo ser consideradas como tecnologías educativas, las que Fainholc (2010) define como

la organización integrada de personas, significados, conceptualizaciones, procedimientos, artefactos simples y/o equipos complejos electronificados, pertinentemente adaptados, a 
ser utilizados para la elaboración, implementación y evaluación de programas, proyectos y materiales educativos que tienden a la promoción del aprendizaje contextuado de un modo libre y creador (p.98).

Es bajo este enfoque que las tecnologías de realidad virtual y realidad combinada pueden ser vistas como medios de desarrollo cognitivo en ambientes educativos, tanto lineales como no lineales (Cubillo, Martín, Castro y Colmenar, 2014) y formales o informales (Echave, Sánchez y Serón, 2016).

Considerando lo anterior y tomando en cuenta las propuestas de la teoría conectivista, en coordinación con las ideas de la alfabetización informacional y la visual literacy, se presentan las siguientes interrogantes: ¿Qué posibilidades educativas nos ofrece la realidad virtual y la realidad combinada desde estas perspectivas? y ¿Qué herramientas tecnológicas de realidad virtual y realidad combinada podrían usarse? Estas preguntas guiarán la reflexión sobre la temática y permitirán sugerir herramientas tecnológicas aplicables a diferentes contextos educativos.

Esta reflexión se fundamenta en la lectura y análisis de diferentes estudios extraídos de diversas bases de datos (EbscoHost, Elsevier, ScienDirect, etc.) y recursos open access tales como Google Académico, ERIC, SciELO, entre otras. En paralelo a estos medios, también se revisaron una serie de documentos contenidos en comunidades académicas 2.0, tales como ResearchGate y Academia.edu. Los documentos fueron seleccionados por su relevancia conceptual, propósito, autenticidad y evolución en el tema (Universidad de Valencia, 2020) centrándose en el periodo del 2000 a la fecha, con especial foco en los últimos cinco años (existen dos excepciones dentro del marco conceptual, que se justifican por su importancia teórica).

En relación con el análisis de la información recopilada para la fundamentación de la reflexión teórica, se empleó el sistema de análisis de texto, que consiste en realizar una lectura detenida, revisar la arquitectura argumental, describir y resumir los documentos. Lo anterior para, posteriormente, contextualizar las ideas que expresan los documentos revisados, formular la reflexión y desarrollar la valoración conclusiva (Florido del Corral, 2020). 


\section{Influencia de la realidad virtual y la realidad combinada en la sociedad de la información}

La sociedad de la información se ha constituido como resultado de un cambio sistemático, veloz y casi imperceptible en diversos procesos sociales. En ella se han adoptado una serie de nuevas tecnologías relacionadas con la información y la comunicación (TIC) que se han incorporado en los ámbitos laborales, culturales, familiares y educacionales. Esto ha hecho que la humanidad esté produciendo la mayor cantidad de conocimiento en su historia, permitiendo al ser humano acceder a esa información sin mayores dificultades.

La incorporación de las TIC también ha producido una serie de transformaciones en los entornos sociales -intercambio masivo de información, uso de herramientas digitales como recursos indispensables para la vida diaria, consideración de la información como bien económico- y cambios político-culturales, producto de la participación ciudadana en la construcción de políticas públicas mediante el uso de medios tecnológicos (Castells, 1996).

Tras estos cambios, ya en la década de los 2000, surgen los denominados dispositivos móviles de comunicación, los cuales introducen una nueva transformación en la dinámica de la sociedad: la información deja de estar a un click de distancia y pasa a estar en el bolsillo de cada usuario/a y, por tanto, a disposición de él/ella en todo momento, hora y lugar (Humanante-Ramos, GarcíaPeñalvo y Conde-González, 2016). En este punto, la sociedad de la información se caracteriza por su alta tecnologización, interconexión, sobreabundancia de datos en todas las áreas y acceso a los recursos informacionales en cualquier instante (Mattelart y Multigner, 2007).

Además de los avances ocurridos a la fecha en la sociedad de la información, ha empezado a masificarse lo que se conoce como la realidad virtual y la realidad combinada, que, si bien no son conceptos nuevos, han adquirido relevancia a medida que la tecnología progresa sistemáticamente (Belli y López, 2008).

Según Gasca-Hurtado, Peña, Gómez-Álvarez, Plascencia-Osuna, y Calvo-Manzano (2015), la realidad virtual es una terminología empleada para describir un entorno tridimensional, el cual es generado por un ordenador y en el que se puede explorar e interactuar con otros individuos. La 
persona se vuelve parte del mundo virtual o está inmersa dentro de este entorno y es capaz de manipular los objetos o realizar una serie de acciones en el entorno diseñado. Esta realidad diseñada es posible catalogarla como realidad virtual inmersiva, semi-inmersiva o inmersiva de proyección, y no inmersiva.

En cambio, la realidad combinada es "una nueva forma de experimentar interacciones en entornos físicos y virtuales a través de un conjunto avanzado de tecnologías de detección y digitalización" (Beroes, 2016, p.1), combinándose para elaborar o reproducir acciones en entornos controlados. Es necesario, para que exista la realidad combinada, que se integren en un solo proceso cinco tecnologías: la detección integrada, los sistemas inalámbricos de control, la manipulación natural, los contenidos digitalizados en función de la realidad y los seis grados de movilidad.

Ambas realidades tienen enfoques distintos: la realidad virtual sumerge a el/la usuario/a en un mundo irreal pero posible, que está diseñado artificialmente y en el que se puede interactuar con personas que comparten ese mismo ambiente; la realidad combinada, en cambio, trata de llevar el mundo físico al mundo virtual en tiempo sincrónico y potenciar la experiencia mediante medios computacionales y digitales (Urquiza, Auria, Daza, Carriel y Navarrete, 2016).

Estos avances técnicos, a pesar de que culturalmente se les asocia más con el mundo de los videojuegos, han tenido un alto impacto en el desarrollo empresarial, al convertirse en herramientas eficaces de visualización de datos específicos y medios de reunión en tiempo real.

Más recientemente, estas innovaciones también han empezado a visualizarse en el mundo educativo redefiniendo los conceptos de e-learning y blended learning (Fabregat, 2012), proponiendo un proceso de enseñanza-aprendizaje fundado en la inmersión de el/la estudiante en una experiencia virtualizada de situaciones laborales o académicas, basadas en experiencias reales de forma controlada y sin riesgos (Jiménez, Villalobos y Luna, 2000). Este tipo de experiencia también es extrapolable al mundo de la medicina y las ingenierías, en las cuales se crean entornos de simulación crítica, validación de las tareas realizadas sin riesgo, sobreimpresión de la información o reducción de errores fatales en el proceso, establecimiento de sistemas guías de resolución de incidencias y optimización de diseños o retroalimentación del proceso ejecutado por el/la usuario/a. 
En cuanto a la gestión de capital humano, se empieza a ver a estas herramientas tecnológicas como medios para lograr seleccionar con eficacia los talentos para las funciones laborales, ya que la realidad virtual y la realidad combinada ayudan a comprobar los saberes profesionales de manera objetiva mediante representaciones de problemas a corregir en los contextos laborales que se desea contratar (Levis, 2006; Cabero y Barroso, 2016), agilizando los procesos de selección y aumentando el número de aciertos al momento de cubrir las vacantes.

Estas herramientas tecnológicas pueden potenciar una variedad de áreas disciplinares, teniendo una especial relevancia aquellas que permiten la masividad, como son el entretenimiento, la comunicación y la educación online. Esto abre paso a sistemas masivos que permiten a las personas experimentar o diseñar estrategias para acceder a una variedad de recursos y fuentes de información que no solo abordan lo racional, sino que permiten explorar la emocionalidad enmarcada en la sociedad de la información (Ricoy, Feliz y Sevillano, 2010). Es en este marco de acción en donde estas tecnologías y su aplicación educativa se vinculan con la teoría del aprendizaje conectivista, propuesta por George Siemens (2004) y Stephen Downes (2006), y las concepciones de alfabetización en información y visual literacy de la Association of College \& Research Libraries (ACRL, 2011).

\section{Conectivismo, alfabetización en información y visual literacy}

Para entender la influencia que pueden tener las tecnologías de realidad virtual y realidad combinada en el desarrollo educativo, es necesario comprender la lógica del conectivismo, que es una teoría del aprendizaje emergente, propuesta por Siemens (2004) y Downes (2006), que integra en una sola mirada los fundamentos del emprendimiento empresarial, el desarrollo de tecnologías y la neurociencia.

El conectivismo visualiza el aprendizaje como un conjunto de conexiones entre nodos que interactúan entre sí, generando redes y ecologías del aprendizaje que permiten que las personas puedan acceder a la información y conocimiento de forma dinámica, a la medida de sus necesidades, con la capacidad de elegir qué aprender en base a múltiples datos digitales. Esto, de 
POSIBILIDADES EDUCATIVAS DE LA REALIDAD VIRTUAL Y LA REALIDAD COMBINADA /

OLIVEROS-CASTRO; NÚÑEZ-CHAUFLEUR

manera de fortalecer saberes previos de manera conectiva, distribuida, colaborativa, cooperativa y normada (Siemens y Colone, 2011).

El enfoque conectivista como planteamiento educativo, dentro del ambiente universitario, es relativamente nuevo y sus premisas desafían muchas de las estructuras ya definidas como canon dentro de la institucionalidad (Casanova, 2012). Es en este punto donde recae la importancia de esta teoría, ya que ofrece perspectivas nuevas para entender la construcción del conocimiento en la era digital (Cabero, 2017) y cómo este conocimiento conectivo (Downes, 2006; Martínez y Martínez, 2017) impacta en el entendimiento y en las acciones de quienes participan en la sociedad de la información.

Junto a lo anterior, esta teoría entrega una perspectiva de cómo las nuevas generaciones posmillennial (Graham, 2018) entienden el aprendizaje como un proceso estrechamente relacionado con las tecnologías 3.0 (Barraza, Ordoñez y Segovia, 2016), la realidad virtual (LópezPellisa, 2015), la realidad ampliada (Prendes, 2015), la realidad combinada (Beroes, 2016), la web semántica (Bernal, Castro, y González, 2017), la gamificación (Sánchez, 2015) y las inteligencias artificiales (Rodríguez y Rosales, 2017).

El impacto educativo del conectivismo se puede vincular con la enseñanza de la alfabetización en información. Esta se entiende como un proceso en constante movimiento que aborda el desarrollo de las destrezas, habilidades y competencias informacionales en las personas, buscando la generación de conocimiento, la autonomía, el procesamiento crítico de las ideas y el aprendizaje a lo largo de la vida (Marzal, 2009). La alfabetización en información (ALFIN) se ve potenciada por las destrezas conectivistas para unir múltiples recursos informacionales, en donde cada persona va reformulando las conexiones a voluntad según su necesidad y requerimientos, en especial en ambientes altamente tecnológicos, en donde los procesos internos y externos de la información buscan cumplir con objetivos educativos institucionales, en concordancia con la integralidad y perfeccionamiento humano de los/as estudiantes.

Esta óptica empleada en la instrucción de contenidos a través de la realidad virtual y realidad combinada, en conexión con la dupla de conectivismo y alfabetización en información, adquiere significado al comprender estos ambientes como fuentes de información multiconectada, que no 
solo requieren de habilidades para la lectura de fuentes escritas de información e interpretación social de contenidos (Agudelo, 2005), sino que, a la vez, requieren de un tercer elemento para que sea efectivo el proceso de aprendizaje con base en las tecnologías. Es aquí donde la alfabetización visual o visual literacy (Freedman, 2019: Koltay, 2019) se vuelve la parte faltante de una triada que da sentido al proceso educativo. Esta comprende la habilidad de descifrar, ajustar y conceder significado a los datos que se presentan en formato de imagen/ícono, extendiendo el constructo de la enseñanza normalmente enfocada en el análisis de los documentos escritos, impresos y digitales (Soto-Grant, 2018). Debes (1969) la define como:

un grupo de competencias vinculadas con la visión propia del ser humano [que] puede[n] ser desarrolladas mediante el ver y al mismo tiempo, el integrar a esa observación, las experiencias sensoriales. El desarrollo de estas competencias es fundamental para el aprendizaje humano normal, puesto que permite que una persona visualmente alfabetizada discrimine e interprete lo visible en las acciones, objetos, símbolos naturales o hechos por el hombre, que se encuentren en su entorno, a través del uso creativo de estas competencias, las cuales lo capacitan para comunicarse con los demás, a través del uso apreciativo de estas, por las cuales es capaz de comprender y disfrutar de las obras maestras de la comunicación visual (p.27).

Es en base a esta definición que la Association of College \& Research Libraries (ACRL, 2011) identifica una serie de competencias relacionadas con el análisis de imágenes: determinar la naturaleza y extensión de los materiales visuales necesarios; encontrar y acceder a las imágenes y los medios visuales necesarios de manera efectiva y eficiente; interpretar y analizar los significados de las imágenes y los medios visuales; evaluar imágenes y sus fuentes; usar imágenes y recursos visuales de forma eficiente; delinear y producir representaciones relevantes; entender las problemáticas éticas, sociales y económicas relacionadas con la creación y el uso de imágenes y medios visuales, y acceso y uso de materiales visuales de manera ética. 
POSIBILIDADES EDUCATIVAS DE LA REALIDAD VIRTUAL Y LA REALIDAD COMBINADA /

OLIVEROS-CASTRO; NÚÑEZ-CHAUFLEUR

\section{Figura 1}

Interacción entre conectivismo, alfabetización en información (ALFIN) y visual literacy

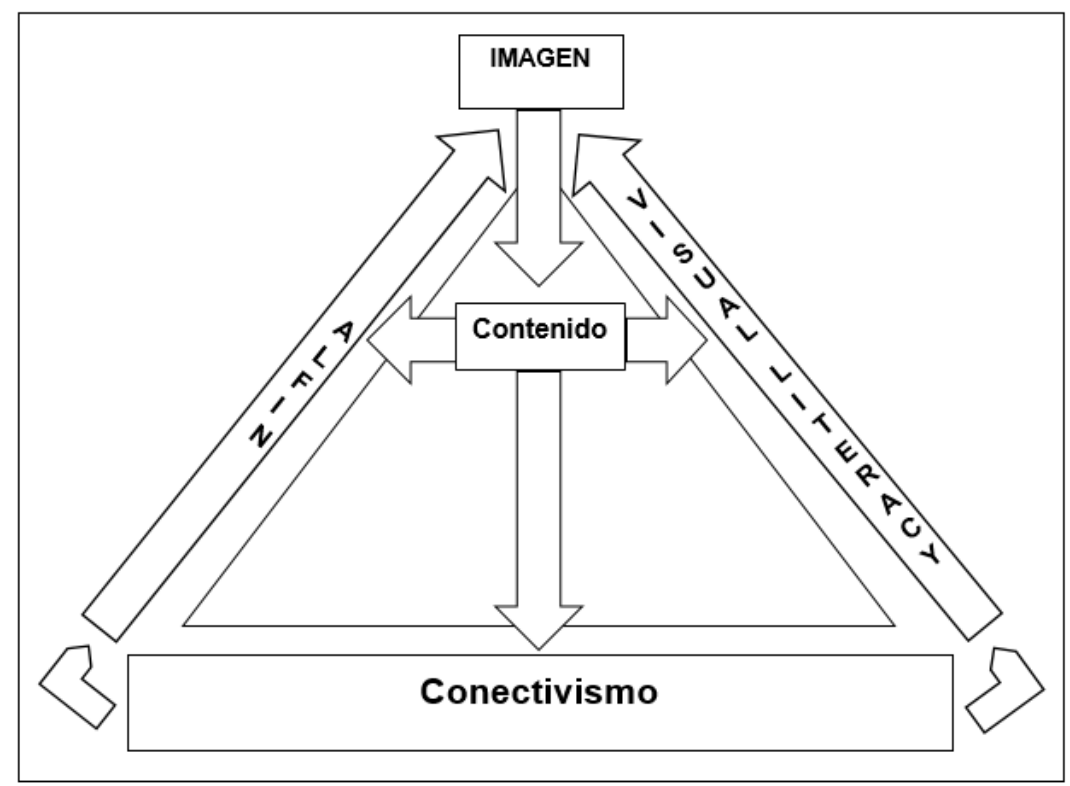

En la conjunción de estos tres elementos, las estrategias de enseñanza que usan la realidad virtual y la realidad combinada se pueden ver reflejadas, por ejemplo, en el empleo de las gafas de realidad virtual con las que se pueden visualizar experiencias profesionales de diferente tipo, destacando los significados simbólicos e informacionales vinculados con los contenidos diseñados previamente para la simulación.

Con esta herramienta, el/la estudiante puede poner en práctica sus saberes profesionales en simulacros inmersivos que aumentan sus capacidades de atención visual, uniendo imágenes ordenadas o aleatorias, las cuales tienen un sentido especifico o múltiple, según el propósito de la actividad. De esta manera, se genera una experiencia y un aprendizaje significativos en el/la estudiante, marcados por el uso de las competencias visuales e informacionales en un solo contenido que, a la vez, se vincula y es resultado de una serie de nodos conectados entre sí que dan sentido y valor educativo a la práctica, sin que esta red sea inmóvil y repetitiva, sino que va 
adecuándose a los objetivos y competencias blandas y profesionales que se desean desarrollar en los/as estudiantes.

Junto a lo anterior, también como método didáctico de enseñanza de contenidos, es posible emplear el espacio de biblioteca como makerspace o espacio de experimentación y laboratorio tecnológico (Buitrago, 2013). En este espacio los/as estudiantes de cualquier carrera, mediante dispositivos de realidad combinada, pueden generar actividades formativas vinculadas con su disciplina, a través de la creación de narraciones de contenidos temáticos o de lectura de textos en $360^{\circ}$ grados, según el requerimiento experiencial de la asignatura.

En términos de impacto educativo, esta sería una aplicación directa de las competencias de la visual literacy y la alfabetización en información, al momento de generar la captura de la realidad y, a la vez, una expresión directa del conectivismo, al generar redes de datos en un espacio de formato análogo, conectándolo e interconectándolo con las fuentes y los medios virtuales de descripción informativa en un todo integrado.

Estos dos ejemplos del potencial educativo de la realidad virtual y la realidad combinada son solo parte de una variedad de ideas que se pueden aplicar no solo en el espacio de la biblioteca, sino también en salones de clases presenciales y virtuales, siendo estos últimos los que ofrecen mayores posibilidades para implementar programas formativos que hagan uso de estos medios y pongan en práctica la lógica descrita con anterioridad en la aplicación de los contenidos temáticos.

\section{Aplicaciones tecnológicas en el ámbito educativo}

A continuación, se presentan algunos dispositivos de realidad virtual y realidad combinada que tienen potencial educativo en la enseñanza (Second Life Data, 2011; QiCanarias.com, 2018; Vive.com, 2020; Versus.com, 2020):

- HTC Vive (realidad virtual): dispositivo de realidad virtual desarrollado por Valve que está disponible desde el 22 de abril de 2015. Este tiene dos pantallas de 2160 x 1200 en total (1080 x 1200 píxeles cada una) con una frecuencia de refresco de 900 Hz. Junto a esto, 
tiene dos dispositivos externos que recogen la posición de los mandos, uno para cada mano. La característica principal de HTC Vive es su sistema de posicionamiento absoluto Lighthouse, el cual puede transformar una habitación en un entorno virtual en el que podremos movernos e interactuar con todo lo que haya en su interior.

- Virtuix Omni (realidad virtual): dispositivo que interactúa con todo el cuerpo. Dispone de una base para el movimiento de las piernas. Este dispositivo se conoce como un andador omnidireccional, capaz de facilitarnos el movimiento y permitirnos andar en un mundo virtual sin movernos en el mundo real, de la misma forma que hacemos en una cinta andadora, pero en todas las direcciones.

- Samsung HMD Odyssey+ (realidad virtual y combinada): este dispositivo tiene una pantalla dual AMOLED de 3,5" que ofrece una resolución combinada de 1440 x 1600p por ojo, además de contar con soporte para sonido espacial de $360^{\circ}$ para simular el audio en 3D, posibilitando la escucha de audio inmersivo con toda claridad desde cualquier ángulo. Dispone de dos cámaras de realidad combinada de Windows, micrófono de doble matriz, sensor IPD, giroscopio, controlador 6DOF e incluye funciones de seguimiento de posición por Bluetooth e Inside-Out, entre otras características.

- Second Life (realidad virtual): es un mundo virtual construido por los/as propios/as participantes. Está compuesto por múltiples servidores en todo el mundo, que se interconectan entre sí para dar estabilidad a la simulación de Second Life. Este mundo virtual se encuentra compuesto por terrenos o sims, que son espacios de 65.000 metros cuadrados equivalentes a un procesador de servidor. Entre algunas de las potencialidades de esta experiencia artificial, se encuentran el poder ser usado como un juego, como un espacio para crear comunidades sociales o empresas y negocios virtuales, fomentar el arte y la cultura mediante la creación de espacios especializados en el tema, como museos y bibliotecas, como red social, para realización de eventos de diferente tipo (deportivos, formales, recreación, etc.) y de educación, mediante la creación de escuelas, universidades y otros espacios para la finalidades instructivas. 


\section{Conclusiones}

Las tecnologías de realidad virtual y combinada son herramientas que ofrecen al mundo formativo las oportunidades de establecer nuevas formas de enseñar los contenidos de la disciplina de manera integral, estableciendo el vínculo directo entre el saber teórico y práctico en un acto virtual, en el que la experiencia se adquiere sin los miedos al error o percances profesionales y/o académicos (Vera, Ortega y Burgos, 2003).

La realidad virtual y la realidad combinada, con el pasar del tiempo, comenzarán a tomar protagonismo a medida que las fuentes de información y medios de acceso a ellas vayan cambiando, dando espacio a formatos en donde el/la usuario/a deberá interactuar de forma directa con los datos, no solo a nivel de cognición, sino a nivel de sensaciones y percepciones. Es en este punto donde la necesidad de filtrar y definir el mundo visual, con todas las conexiones que la realidad virtual y la realidad combinada pueden generar, requerirá cada vez más de la integración y posicionamiento del conectivismo, la alfabetización en información y la visual literacy, como catalizadores de los cambios y medios de control informacional en esos entornos.

La realidad virtual y la realidad combinada, por tanto, son herramientas para el descubrimiento y la presentación de datos complejos en forma dinámica y comprensible para el/la estudiante, quien está sujeto/a al enfoque cambiante de la educación y de las imágenes que estas presentan delante de las pantallas. Es aquí donde las didácticas de aprendizaje deben mejorar sus técnicas de adquisición de conocimiento, potenciándolas mediante estrategias integrales como el visual thinking o pensamiento visual, que consiste en entender los patrones o ideas que son parte de una imagen, fuente de información o nodo conectivo, que se vuelven un elemento clave al momento de establecer un punto de referencia entre la realidad, la realidad virtual y la realidad combinada (Beroes, 2016).

Estos dispositivos digitales ayudan en la síntesis de los procesos de enseñanza-aprendizaje, permitiendo que el/la estudiante los asocie con el escuchar, el sentir y el ver, estableciendo finalmente un mapa lógico, emocional y perceptual que convierte el contenido enseñado en un aprendizaje significativo (Soto-Grant, 2018). 
A su vez, el proceso de adquisición de las competencias informacionales, las habilidades de interpretación visual y la comprensión de las ideas del conectivismo, requieren de un periodo prolongado que, al finalizarse, permite al/la estudiante adecuarse a los cambios tecnológicos y educacionales que surgieran.

Es necesario mencionar que, aunque la realidad virtual y la realidad combinada representan una oportunidad, existe una serie de falencias en los sistemas educativos que impiden la inserción de estas tecnologías, comenzando por el desconocimiento parcial o total del potencial para los/as docentes y estudiantes, pasando por la falta de cooperación entre las bibliotecas y las iniciativas docentes; desembocando en la falta de apoyo institucional por la incomprensión sobre la temática en cuestión. Esta situación provoca que el proceso de enseñanza-aprendizaje empleando estas tecnologías y enfoques no sea fácilmente desarrollable y evaluable durante un periodo de tiempo extendido.

\section{Referencias}

Agudelo, A. (2005). Modelo de contexto para realidad aumentada. Revista Universidad EAFIT, 41(138), 44-64. Recuperado de https://cutt.ly/Lt9vhz1

Association of College \& Research Libraries (ACRL) (2011). ACRL Visual literacy competency standards for higher education. Recuperado de https://cutt.ly/Ft9v40Z

Barraza, F., Ordoñez, H., y Segovia, E. (2016). Diseño de una plataforma de colaboración para investigación científica basada en e-Science 3.0. Campus Virtuales, 5(1), 92-98. Recuperado de https://cutt.ly/Zt9bisO

Belli, S., y López, C. (2008). Breve historia de los videojuegos. Athenea Digital. Revista de Pensamiento e Investigación Social, (14), 159-179. Recuperado de https://cutt.ly/ot9bbc4 
REVISTA SABERES EDUCATIVOS, Nº 5, JULIO-DICIEMBRE 2020

Bernal, D., Castro, A., y González, J. (2017). Web semántica, más de una década de su aparición. Puente, 8(1), 61-69. DOI: http://dx.doi.org/10.18566/v8n1.a07

Beroes, M. (2016). 5 avances tecnológicos aplicados a la realidad combinada. Recuperado de https://cutt.ly/it3aNMM

Buitrago, R. (2013). Estado del arte: Realidad aumentada con fines educativos. Escuela Colombiana de Carreras Industriales. ECCI, 2(3), 50-59. Recuperado de https://cutt.ly/it9nkm0

Cabero, J. (2017). La formación en la era digital: ambientes enriquecidos por la tecnología. Revista Gestión de la Innovación en Educación Superior, 2(1), 34-53. Recuperado de https://cutt.ly/at9vSBV

Cabero, J., y Barroso, J. (2016). Posibilidades educativas de la Realidad Aumentada. Journal of New Approaches in Educational Research, 5(1), 46-52. DOI: 10.7821/naer.2016.1.140

Casanova, M. (2012). El diseño curricular como factor de calidad educativa. REICE. Revista Iberoamericana sobre Calidad, Eficacia y Cambio en Educación, 10(4), 6-20. Recuperado de https://cutt.ly/Dt9nMug

Castells, M. (1996). La era de la información Vol. I: La sociedad Red. Madrid: Alianza Editorial.

Cubillo, J., Martín, S., Castro, M. y Colmenar, A. (2014). Recursos digitales autónomos mediante realidad aumentada. RIED. Revista Iberoamericana de Educación a Distancia, 17(2), 241274. Recuperado de https://cutt.ly/Nt9mtCG

Debes, J. (1969). The loom of visual literacy: An overview. Audiovisual Instruction, 14(8), 25-27.

Downes, S. (2006). Learning Networks and Connective Knowledge. DOI: 10.4018/978-1-60566729-4.ch001

Echave, A., Sánchez, M. y Serón, F. (2016). Un escenario creativo para la educación científica mediante la Realidad Ampliada. Revista de investigación en educación, 2(14), 240-246. Recuperado de https://cutt.ly/it9mWMI 
POSIBILIDADES EDUCATIVAS DE LA REALIDAD VIRTUAL Y LA REALIDAD COMBINADA /

OLIVEROS-CASTRO; NÚÑEZ-CHAUFLEUR

Fabregat, R. (2012). Combinando la realidad aumentada con las plataformas de e-elearning adaptativas.Enl@ce: Revista Venezolana de Información, tecnología y conocimiento, 9(2), 69-78. Recuperado de https://cutt.ly/Nt9mDUX

Fainholc, B. (2010). Diccionario práctico de tecnología educativa. REDHECS. Revista Electrónica de Humanidades, Educación y Comunicación Social, 9(5), 162-167. Recuperado de https://cutt.ly/1t9m8uw

Florido del Corral, D. (2020). Esquema modelo para realización de análisis/comentarios de textos. Recuperado de: https://cutt.ly/NyNkz4h

Freedman, K. (2019). Visual Culture and Visual Literacy. The International Encyclopedia of Art and Design Education, 1-10. DOI: https://doi.org/10.1002/9781118978061.ead079

Gasca-Hurtado, G., Peña, A., Gómez-Álvarez, M., Plascencia-Osuna, Ó., y Calvo-Manzano, J. (2015). Realidad virtual como buena práctica para trabajo en equipo con estudiantes de ingeniería. RISTI-Revista Ibérica de Sistemas e Tecnologias de Informação, (16), 76-91. DOI: http://dx.doi.org/10.17013/risti.16.76-91

Gay, A., y Ferreras, M. (2016). La educación tecnológica: aportes para su implementación. México D.F.: Editorial Brujas.

Graham, L. (2018). Generation Z Goes to Law School: Teaching and Reaching Law Students in the Post-Millennial Generation. University of Arkansas at Little Rock Law Review, 41(1), 29-95. Recuperado de: https://cutt.ly/zt3oKiG

Humanante-Ramos, P., García-Peñalvo, F., y Conde-González, M. (2016). PLEs en contextos móviles: Nuevas formas para personalizar el aprendizaje. IEEE VAEP - RITA, 4(1), 33-39. Recuperado de https://cutt.ly/5t3oEsA

Jiménez, A., Villalobos, M., y Luna, E. (2000). Cuándo y cómo usar la Realidad Virtual en la Enseñanza. Revista Iberoamericana de Informática Educativa, (16), 26-36. Recuperado de https://cutt.ly/yt3piAV 
REVISTA SABERES EDUCATIVOS, Nº 5, JULIO-DICIEMBRE 2020

Jiménez, J. (2014). Modelo de un Sistema Organizacional Conectivista. Recuperado de https://cutt.ly/3t3pvb1

Koltay, T. (2019). Visual Literacy. The International Encyclopedia of Media Literacy, 1-6. DOI: https://doi.org/10.1002/9781118978238.iem10242

Levis, D. (2006). ¿Qué es la realidad virtual? Recuperado de https://cutt.ly/vt3p2Mp

López-Pellisa, T. (2015). Patologías de la realidad virtual: Cibercultura y ciencia ficción. Madrid: Fondo de Cultura Económica.

Martínez, F., y Martínez, A. (2017). Fundamentos del aprendizaje en red desde el conectivismo y la teoría de la actividad. Revista Cubana de Educación Superior, 35(3), 98-112. Recuperado de https://cutt.ly/ot3afwS

Marzal, M. (2009). Evolución conceptual de la alfabetización en información a partir de la alfabetización múltiple en su perspectiva educativa y bibliotecaria. Investigación bibliotecológica, 23(47), 129-160. Recuperado de https://cutt.ly/1t3amKq

Mattelart, A. y Multigner, G. (2007). Historia de la sociedad de la información. Buenos Aires, Barcelona, México: Paidós.

Prendes, C. (2015). Realidad aumentada y educación: Análisis de experiencias prácticas. Pixel-Bit. Revista de Medios y Educación, (46), 187-203. DOI: 10.12795/pixelbit.2015.146.12

QiCanarias.com. (2018). Virtuix Omni Plataforma + Sensores Pies + Softwares con juegos. Recuperado de: https://cutt.ly/Pyuarf8

Ricoy, M., Feliz, T. y Sevillano, M. (2010). Competencias para la utilización de las herramientas digitales en la sociedad de la información. Educación xxl, 13(1), 199-219. DOI: https://doi.org/10.5944/educxx1.13.1.283

Rodríguez, J. y Rosales, J. (2017). Extracción de conocimiento mediante la aplicación de inteligencia artificial a la información espacial. Mapping, (186), 40-47. Recuperado de https://cutt.ly/bt9bNxd 
POSIBILIDADES EDUCATIVAS DE LA REALIDAD VIRTUAL Y LA REALIDAD COMBINADA /

Sánchez, F. (2015). Gamificación. Education in the Knowledge Society (EKS), 16(2), 13-15. DOI: http://dx.doi.org/10.14201/eks20151621315

Second Life Data. (2011). ¿Qué es Second Life? ¿Para que sirve Second Life? ¿Es un juego? Recuperado de https://cutt.ly/0yupkS6

Siemens, G. (2004). Conectivismo: Una teoría de aprendizaje para la era digital. Recuperado de https://cutt.ly/rt3dpDL

Siemens, G. y Colone, G. (2011). Special issue - connectivism: Design and delivery of social networked learning. International Review of Research in Open and Distance Learning, 12(3), 1-4. Recuperado de https://cutt.ly/ut3dvGB

Soto-Grant, A. (2018). Habilidades y estrategias didácticas necesarias para la alfabetización visual en educación preescolar. Revista Electrónica Educare, 22(3), 1-17. DOI: 10.15359/ree.223.2

Tobón, S., Guzmán, C., Hernández, J., y Cardona, S. (2015). Sociedad del conocimiento: estudio documental desde una perspectiva humanista y compleja. Paradigma, 36(2), 7-36. Recuperado de https://cutt.ly/Ft3gxRl

Universidad de Valencia. (2020). Competencias informacionales nivel medio: 2.3. Criterios para seleccionar la fuente de información adecuada. Recuperado de: https://cutt.ly/jyNkovk

Urquiza, L., Auria, B., Daza, S., Carriel, F. y Navarrete, R. (2016). Uso de la realidad virtual, en la educación del futuro en centros educativos del Ecuador. Journal of Science and Research. Revista Ciencia e Investigación, 1(4), 26-30. DOI: https://doi.org/10.26910/issn.25288083vol1iss4.2016pp26-30

Vera, G., Ortega, J. y Burgos, M. (2003). La realidad virtual y sus posibilidades didácticas. Etic@net, 2(2), 1-17. Recuperado de https://cutt.ly/Xt3bm00

Versus.com. (2020). Samsung HMD Odyssey. Recuperado de: https://cutt.ly/vyuavEL

Vive.com. (2020). Serie Cosmos. Recuperado de: https://cutt.ly/eyupBlx 